\title{
Negative capacitance and instability at electrified interfaces: Lessons from the study of membrane capacitors
}

\author{
M.B.Partenskii, P.C.Jordan \\ Department of Chemistry, MS-015 \\ Brandeis University \\ PO Box 549110 \\ Waltham, MA 02454-9110, USA
}

Received September 14, 2004

\begin{abstract}
Various models leading to predictions of negative capacitance, $C$, are briefly reviewed. Their relation to the nature of electric control is discussed. We reconfirm that the calculated double layer capacitance can be negative under $\sigma$-control - an artificial construct that requires uniform distribution of the electrode surface charge density, $\sigma$. However, only the total charge $q$ (or the average surface charge density $\bar{\sigma}$ ) can be experimentally fixed in isolated cell studies $(\boldsymbol{q}$-control). For those $\sigma$ where $C$ becomes negative under $\sigma$-control, the transition to $q$-control (i.e. relaxing the lateral change density distribution, fixing its mean value to $\sigma$ ) leads to instability of the uniform distribution and a transition to a non-uniform phase. As an illustration, a "membrane capacitor" model is discussed. This exactly solvable model, allowing for both uniform and inhomogeneous relaxation of the electrical double layer, helps to demonstrate both the onset and some important features of the instability. Possibilities for further development are discussed briefly.
\end{abstract}

Key words: electrochemical interfaces, instabilities and phase transitions, electric double layers, capacitance, membrane electroporation

PACS: 68.35.Rh, 68.35.Md, 82.45.Mp, 82.45.Rr, 82.45.Uv, 68.08.-p, 73.30. $+y$

\section{Introduction}

The question of the admissible sign of the differential capacitance at charged interfaces and its relation to thermodynamic stability has a long and dramatic history. It emerged in early 1970s with the so-called Cooper-Harrison catastrophe [7], an apparent prediction of $C<0$ for "dipolar capacitors" - lattices of reorientable dipoles between conductive plates. Soon thereafter, similar anomalies were predicted for some ionic double layer models [3,12]. In the mid-1980s interest in this problem was 
renewed due to predictions of $C<0$ for microscopic "relaxing gap capacitor" (RGC) models (see $[22,26,27,30]$ and references therein) which accounted for the metal electron contributions and the corresponding relaxation of the "electronic plate" of the interfacial capacitor with charging. It is accepted that $C$ must be strictly positive for open " $\phi$-controlled" systems, where the electric cell is connected to a source of controllable voltage [15]. However, it was shown that $C<0$ is possible as a stable state of an isolated RGC if electric control is maintained by a macroscopically uniformly distributed surface charge density $\sigma$, so-called $\sigma$-control (see $[10,22,26]$ for review). Prior to this discovery, an attempt had been made to prove, on general statistical-mechanical grounds, that for an equilibrium $\sigma$-controlled system $C$ must be strictly positive [4]. Somewhat later improved analysis $[2,20,21,24,27]$ showed the model [4] does not forbid negative $C$ values. For both ionic and "relaxing gap capacitor" models, which together cover a very wide range of interfacial phenomena, the equilibrium capacitance under $\sigma$-control can be negative. This is true for both the "compact layer" capacitance and the total double layer capacitance, including the "diffuse" layer contributions $[22,26]$.

Here we show that though admissible for $\sigma$-control, which implies a uniform charge density on the "electrode", negative EDL capacitance is not possible for a real isolated system, where uniformity of $\sigma$ is not enforceable and only the total electrode charge can be fixed. In other words, we show that in $\sigma$ domains where $C$ is negative under $\sigma$-control, the system is unstable with respect to transition to an inhomogeneous state with nonuniform lateral distributions of the electrode charge density and of mobile electrolyte ions. To demonstrate this anomaly, we discuss a transparent "membrane capacitor" model, which exhibits a $C<0$ domain presuming uniformity (an elastic slab with plane surfaces and uniform surface charge) while becoming unstable in this domain if non-uniform surface charge distributions and membrane deformations are considered.

This result addresses questions raised recently $[6,11]$ with respect to the meaning and physical reality of $C<0$ for the isolated capacitor. Our analysis suggests that a model demonstrating this anomaly under $\sigma$-control can be used to study transition to an inhomogeneous interfacial state under $q$-control.

\section{Control of electrified interfaces - theory and experiment}

1. Experimental study of the electrical double layer (EDL) at electrochemical interfaces is usually conducted under "potential" $(\phi-)$ control, where electrodes are connected to a voltage source. Changing the applied voltage in increments $\Delta \phi$ leads to corresponding changes of the electrode charge, $\Delta q$. Similarly, controlled modulation of the voltage, $\phi=\phi(t)$, results in charge modulation measurable by impedance techniques. Connection to a potentiostat, required to maintain $\phi$-control, results in an open system, which is treated by grand canonical methods. 
The differential capacitance (per unit area) is then defined by the derivative

$$
C_{\phi}=\partial_{\phi} \bar{\sigma}
$$

where the average surface charge density is $\bar{\sigma}=q / A$ and $A$ is the surface area of the electrode. The form of equation 1 is a typical response function

$$
\varkappa_{\mathrm{F}}=\partial_{\mathrm{F}} X
$$

where $\mathrm{F}$ is the external parameter ("force") and $X$ the conjugate intensive variable.

2. The electrical properties of interfaces can equally well be studied by controlling the electrode charge, $q$. In $q$-control the measured quantity is the corresponding potential $\phi$. Charge can be regulated by connecting the electrodes to a battery for brief periods of time $\Delta t$, measuring the current $j$, with the charge increments found by $\Delta q=j \Delta t$. With $q$ fixed, the resultant $\phi$ is measured in an isolated system, i.e. a canonical ensemble.

The corresponding response function, analogous to equations 1 and 2 is the inverse differential capacitance

$$
C_{q}^{-1}=\partial_{\bar{\sigma}} \phi
$$

Obviously, $q$-control is a synonym for $\bar{\sigma}$-control: fixing the total charge $q$ is equivalent to fixing the average surface charge density $\bar{\sigma}$. The thermodynamic potentials of the expanded ( $\phi$-controlled ) system, $A_{\phi}$, and the isolated $(q-$ controlled) system, $A_{q}$, are related by the Legendre transformation,

$$
A_{\phi}(\bar{\sigma}, \phi)=A_{q}(\bar{\sigma})-q \phi
$$

3. Most calculations of the electric double layer (EDL) assume that the electrode, often described as a charged flat wall, has a uniform, fixed charge density. This is effectively $\sigma$-control, which is generally not equivalent to $q$ - (or $\bar{\sigma}_{-}$) control. The terms are interchangeable only if the equilibrium surface charge density is uniform on a scale exceeding atomic dimensions, i.e. if $\sigma=\bar{\sigma}=$ const. A counter-example is the one where the optimized local charge density is nonuniform in the electrode plane, $\sigma=\sigma\left(r_{s}\right) \neq \bar{\sigma}\left(r_{s}\right.$ is the radius vector in the electrode plane), reminiscent of "charge density wave" states in an electron plasma. In practice there is no way to control the local charge density; in isolated systems only the total charge can be constrained externally. $\sigma$-control is a purely theoretical construct; its predictions must be tested to determine if a uniform surface charge density and its corollary, a laterally-uniform ionic distribution, correspond to a real equilibrium state. 


\section{Admissible sign of the differential capacitance}

\section{1. $\phi$-control: the open system}

General thermodynamic [14] and statistical-mechanical [18] treatments of electrified interfaces show that, under $\phi$-control, differential capacitance must be strictly positive. In our view attempts to circumvent this restriction $[2,31,32]$ have somewhat misinterpreted the concept of potential control [26,27].

Simple models $[9,10,22,26,27]$ demonstrate that "equilibrium" states leading to predictions of $C<0$ under $\phi$-control are inevitably unstable (and therefore fictitious), corresponding to local maxima in the thermodynamic potential $A_{\phi}$. Typically, a different locally stable state (or states) for which $C>0$ should exist for the same voltage $\phi$. Thus, predictions of $C<0$ in open systems must be tested with respect to stability and to the existence of alternative, stable states. Recent analysis [6] has compared two theoretical approaches to the modeling of the EDL: (1) Monte-Carlo treatment of EDL under $\sigma$-control and (2) Density-Functional Theory for the open system $(\phi$-control). Both approaches predict $C<0$. While this prediction can be valid in the framework of the first approach (see e. g. $[26,27]$ and the following discussion), it must be carefully reexamined for an open system.

Near a critical voltage $\phi_{\mathrm{cr}}$, defined by $C^{-1}\left(\phi_{\mathrm{cr}}\right)=0$, the system becomes unstable. The transition to a new state is accompanied by charge flow from the potentiostat to the electrodes, a sort of electrical "breakdown" (see $[9,10,22]$ for more details), which would be a unique path to phase transformation assuming lateral uniformity. However, as discussed below, the transition can also involve formation of a laterally non-uniform phase accompanied by nonuniform redistribution of the electrode charge density $\sigma(\rho)$.

As the requirement that $C_{\phi}>0$ is now generally accepted, we turn to treating isolated systems. The sense of the upcoming discussion is already implicit, once having recognized that the first of the two aforementioned instabilities is forbidden by the very definition of " $q$-control" since, once $q$ is fixed, electric contact with the potentiostat must be interrupted. Can $C$ be negative under this constraint? Since the admissible sign of $C$ in isolated systems has almost always been analyzed in $\sigma$-control terms, we first consider this case and postpone discussion of the more general $q$-control.

\section{2. $\sigma$-control in the isolated system}

\subsubsection{Primitive models of electrolytes}

Interest in the admissible sign of $C$ in the theory of the diffusive layer was stimulated by work of Blum, Lebowitz and Henderson [4]. They tried to provide a rigorous restriction on the sign of $C$ for "primitive ionic models": charged hard ions in a uniform dielectric medium between two rigid, uniformly charged walls. The corresponding Hamiltonian is quite generally [24]

$$
H(\sigma,\{\mathbf{R}\})=\frac{\sigma^{2} d}{2 \varepsilon \varepsilon_{0}}-\sigma f(\{\mathbf{R}\})+H^{\prime}(\{\mathbf{R}\}),
$$


where $\{\mathbf{R}\}$ refers to a particular configuration of the system (charge coordinates, dipolar orientations, etc.). The first term describes a direct interaction between the charged walls, with $d$ the inter-wall distance. $\sigma f(\{\mathbf{R}\})$ accounts for interaction between the electrolyte and the electrode field (the physical significance of $f$ will be clear shortly) and $H^{\prime}$ is a $\sigma$ independent interaction energy. The potential drop between the charged plates is

$$
\phi(\sigma)=\frac{\sigma d}{\varepsilon \varepsilon_{0}}+\langle f\rangle
$$

where

$$
\langle(\ldots)\rangle=\frac{\int \mathrm{e}^{-\beta H(\sigma,\{\mathbf{R}\})}(\ldots) \mathrm{d} \Omega}{\int \mathrm{e}^{-\beta H(\sigma,\{\mathbf{R}\})} \mathrm{d} \Omega}
$$

is a canonical average with integration over the system's configurational space, $\Omega$, and $\beta=1 / k T$. Equation 6 reveals the meaning of $f:\langle f\rangle$ is the potential drop induced in the electrolyte by the field of the charged plates. It arises from redistribution of free (ionic) charges shielding the applied field, and from repositioning of the bound charges (the reorientation of molecular dipoles).

For Hamiltonians of the type of equation 5 the capacitance satisfies the general condition $[24]$

$$
C^{-1}=\frac{d}{\varepsilon \varepsilon_{0}}-\frac{A}{k T}\left(\left\langle f^{2}\right\rangle-\langle f\rangle^{2}\right),
$$

leading to a simple and self-evident result:

$$
C^{-1} \leqslant \frac{d}{\varepsilon \varepsilon_{0}} .
$$

Equation 8 simply means that redistribution of free charges and molecular polarization induced by the electric field in the electrolyte reduce the potential drop between the electrodes and increase the capacitance $C$, a condition that places no constraints on the sign of $C$ under $\sigma$-control. One should note here that if the distance $d \gg \lambda_{\mathrm{D}}$, the characteristic Debye length in the electrolyte, then the total inverse capacitance of the cell splits into two independent double layer contributions belonging to two "electrodes":

$$
C^{-1}=C_{1}^{-1}+C_{2}^{-1}
$$

If the sign of the total cell capacitance $C$ is unrestricted under $\sigma$-control, this is even more true for the individual double layer contributions, $C_{1}$ and $C_{2}$.

Equation 7 (equation 24 of [24]) was derived in a study of a "dipolar capacitor" ("DC"), a lattice of point dipoles embedded between the plates of a parallel-plate capacitor, a model often used for analyzing a compact layer at metal-solvent interfaces. For the DC

$$
f=f_{\mathrm{DC}}=-\frac{1}{\varepsilon_{0}} P_{z}=\frac{1}{A \varepsilon_{0}} \sum_{i} p_{i, z}
$$

is the potential drop corresponding to an arbitrary configuration of the molecular dipoles with $P_{z}$ the average surface density of the dipolar moment in the lattice 
and $p_{i, z}$ the projection of the individual dipole moment normal to the surface of the lattice.

If interaction between the charged walls (the first contribution to the Hamiltonian, equation 5) were properly included in the Hamiltonian of the primitive ionic model [4], it would also lead to equation 7 (see p. 68 of [24]) with

$$
f=f_{\text {ion }}=-\frac{1}{\varepsilon_{0}} \sum q_{i} z_{i}
$$

where $q_{i}$ is the charge of the $i$-th ion and $z_{i}$ is its distance from the charged wall positioned at $z=0$. Equation 7 and its analogs have been repeatedly derived and discussed $[2,21,27]$; they hold for any model in which the electrodes are treated as hard charged walls with distinctly separate regions occupied by electrode and electrolyte. With these restrictions the interaction between the electrode and the electrolyte can quite generally be described by a contribution $\sim \sigma \sum q_{i} z_{i}$ where the summation includes both the mobile ionic and the molecular multipole charges [27]. Such constraints clearly exclude "polarizable" models, those explicitly treating molecular electronic polarizability, electron density penetration into regions occupied by electrolyte, etc.

\subsubsection{Relaxing gap capacitors (RGC)}

Immobility of the charged "plates" in primitive models does not account for another important phenomenon, possible displacement of the "electronic plate" of interfacial capacitors and of the equilibrium positions of the electrolyte species in contact with the electrode, in response to charging $[22,26,27,30]$. These effects are effectively illustrated by the "relaxing gap capacitor" metaphor, which emphasizes the dependence of the effective gap $d$ on charging. The potential drop can be quite generally represented as

$$
\phi=\frac{1}{\varepsilon \varepsilon_{0}} \sigma d(\sigma)
$$

where $d(\sigma)$ is the effective separation between the "plates" of the capacitor associated with the "centers of mass" of two microscopic charge distributions (see $[10,22,30]$ for review). We assume a uniform dielectric background $\varepsilon$ between the plates, in the volume occupied by free charges. For models using a non-uniform background, typical of unified models used to account for both the "inner" (or Helmholtz) and the diffuse layers [5], the expression is more complex and must also account for the distributions of the bound (polarization) charges. However, these details are not essential; they are omitted here.

For the electrode-electrolyte interface the effective separation is

$$
d(\sigma)=z_{i}(\sigma)-z_{i, e}(\sigma)
$$

where

$$
z_{i, e}=\frac{\int \rho_{i, e}^{\sigma} z \mathrm{~d} z}{\sigma}
$$


with $z$ the coordinate normal to the electrode surface. The inverse differential capacitance for the RGC is

$$
\left(\frac{1}{\varepsilon \varepsilon_{0}} C\right)^{-1}=(\sigma d(\sigma))_{\sigma}^{\prime}=d(\sigma)+\sigma d(\sigma)_{\sigma}^{\prime} .
$$

Dependence of $d$ on $\sigma$, a general feature of practically all double layer models, implies that $C$ is dependent on $\sigma$ (or on the applied voltage). The "plate" displacement that contributes to the variation of $d$ not only reflects a shift of charge density profiles, but is more a consequence of shape variation [26]. Quite typically, there is always a $\sigma$ range in which the charging decreases the effective gap. Elastic compression of the lipid membrane by electric stress $[8,23,25]$ and response of the Gouy-Chapman-Stern (GCS) diffuse layer to the charging [5] are two representative examples.

In a range of $\sigma$ where the effective gap contracts with the charging, $d^{\prime}(\sigma)<0$ and $C_{\sigma}$ can be negative if

$$
\Delta \sigma \cdot d-\sigma \cdot \Delta d<0
$$

This inequality means that a potential increase due to a change of $\sigma$ is overwhelmed by its decrease due to gap contraction. A number of electromechanical and microscopic models $[10,22,26,27]$ show that negative capacitance (NC) under $\sigma$-control is compatible with system stability. For instance, for every fixed $\sigma$ (including the domain where $C<0$ ) the equilibrium gap of the elastic capacitor is defined by a stable balance between elastic and electrostatic forces. Similarly, density functional minimization led to a $\mathrm{NC}$ at metal-electrolyte interfaces due to relaxation of the "electronic plate" of the capacitor $[9,10,13]$.

Previous work $[10,22,28]$ showed that accounting for "electronic plate" relaxation in combination with traditional GCS and similar models typically leads to negative $C$ domains even though the GCS model itself (as does any other "local" statistical model where ionic concentrations are local functions of the potential) does not lead to such an anomaly [10]. Thus, while we agree that finding $C<0$ under $\sigma$-control must be both common and important for ionic models of electrolytes [11], it is not a necessary condition for the appearance of this anomaly. If other relaxation mechanisms are taken in account a NC domain may arise even if the ionic contribution is positive.

\section{3. $q$-control in the isolated system}

As discussed, $\sigma$-control is an artificial construct. Nevertheless, charges are normally distributed uniformly in the plane of the electrode and the charge $q$ naturally yields a uniform charge density $\sigma$, in which case $\sigma$ - and $q$-control are identical. However, it is possible that under special conditions a non-uniform distribution of charge in the electrode plane becomes energetically preferable. Under these conditions the $\sigma$-control metaphor implies that artificial restraints (forces) were applied to the surface charges to enforce uniformity of $\sigma$. Elimination of these artificial restraints would result in transition to an inhomogeneous state. We will show this and its relation to the sign of the capacitance by considering an undulating membrane capacitor, an 
exactly solvable model that contains many features common to real EDLs, which is thus generally useful for discussing double layer behavior under critical conditions.

For a membrane capacitor under potential control the onset of instability results from the steep increase of the electrostatic force $\sim \phi^{2} / h^{2}$ where $\phi$ is the applied potential and $h$ is the membrane thickness. This arises because the system is open: as $h$ decreases, the thinning leads to charge transfer between a battery and the plates of the capacitor required to maintain the fixed value of the potential drop $\phi \sim \sigma h=$ const. Mathematically, charge transfer is controlled by the term $-q \phi$ relating the thermodynamic potentials of the open and isolated capacitor (equation 4). Thus, both the charge density and the attractive force increase unrestrainedly as the plates approach one another. At a critical voltage, no increase of the elastic repulsive force can compensate for the catastrophic increase of the electrostatic attraction, leading to the potential-driven instability first noted by Crowley [8].

In an isolated system this pathway to instability is forbidden. When isolated, the total plate charge is fixed and uniform system thinning cannot increase the attractive force, which remains constant, $\sim \sigma^{2}$. Instability can only result from nonuniform redistribution of the charge density in the plane of the membrane capacitor with an associated inhomogeneity in $h$. Thus we are led to consider the possible instability of an isolated flexible membrane capacitor. For $\phi$-control we solved this problem for electrolyte charge distributions that satisfy the Poisson-Boltzmann equation [23]. Here we consider a simplified example, a capacitor in contact with a "perfect conductor," i.e. $\varepsilon_{\text {solvent }}=\infty$, so that the membrane surfaces are isopotentials, a reliable approximation even for dilute electrolytes [23].

Consider the parallel-plate membrane capacitor with its midplane at $z=0$ and an unperturbed thickness (when $q=0$ ) equal to $h_{0}$. Charging the membrane to charge density $\sigma$ yields an electrostatic pressure which gives rise to membrane compression. Introducing the thinning coefficient $\alpha=\bar{h} / h_{0}$, with $\bar{h}=\bar{h}(\sigma)$ the average thickness of the compressed membrane, the total energy of the uniform slab is

$$
W_{0}=W_{d, 0}+W_{e, 0}, \quad \text { where } \quad W_{d, 0}=\frac{1}{2} K_{\mathrm{s}}(\alpha-1)^{2} \quad \text { and } \quad W_{e, 0}=\frac{\bar{h}}{2 \varepsilon \varepsilon_{0}} \sigma^{2}
$$

the two terms are the harmonic approximation to the deformation (stretchingcompression) energy and the electrostatic energy respectively, $K_{\mathrm{s}}$ is the stretching modulus and the index " 0 " refers to a uniformly deformed membrane. The equilibrium membrane thickness $\bar{h}(\sigma)=h_{0} \alpha(\sigma)$ is found from the condition $\partial_{\alpha} W=0$ leading to the thinning coefficient

$$
\alpha(\sigma)=1-\frac{1}{3}\left(\frac{\sigma}{\sigma_{\mathrm{cr}}}\right)^{2}
$$

where

$$
\sigma_{\mathrm{cr}}=\sqrt{\frac{2 K_{\mathrm{s}} \varepsilon \varepsilon_{0}}{3 h_{0}}}
$$


which is interpreted in what follows. The transmembrane potential drop and the corresponding inverse differential capacitance are

$$
\phi=\frac{1}{\varepsilon \varepsilon_{0}} \sigma \bar{h}(\sigma)=\frac{1}{\varepsilon \varepsilon_{0}} h_{0} \sigma\left[1-\frac{1}{3}\left(\sigma / \sigma_{\mathrm{cr}}\right)^{2}\right]
$$

and

$$
C_{\sigma}^{-1}(\sigma)=\frac{\mathrm{d} \phi}{\mathrm{d} \sigma}=C_{0}^{-1}\left[1-\left(\sigma / \sigma_{\mathrm{cr}}\right)^{2}\right]
$$

where

$$
C_{0}^{-1}=\frac{1}{\varepsilon \varepsilon_{0}} h_{0}
$$

is the inverse capacitance of a capacitor with the fixed gap $h_{0}$. Equation 17 reveals the meaning of $\sigma_{\mathrm{cr}}$. It is the charge density where the differential capacitance $C_{\sigma}$ becomes infinite; $C_{\sigma}$ is negative for $|\sigma|>\sigma_{\mathrm{cr}}$. The corresponding membrane thickness is

$$
\bar{h}\left(\sigma_{\mathrm{cr}}\right)=\frac{2}{3} h_{0},
$$

at $\sigma=\sigma_{\text {cr }}$ the membrane has thinned by $\sim 33 \%$, a value typical of "relaxing gap" capacitor models $[13,22,23,26,27,29]$. The membrane is stable relative to virtual uniform compression (thinning) under $\sigma$-control (i.e. assuming a uniform surface charge density) for all $\sigma$ including the range where $C<0$. This is verified from equation 13:

$$
\left.\partial_{\alpha \alpha}^{2} W\right|_{\sigma}=\frac{K_{\mathrm{s}}}{\alpha}>0 .
$$

We now focus on the energy change, $\Delta W$, for an isolated membrane capacitor in response to a small charge increment, $\Delta \sigma$, assuming $\sigma$-control. Using the relation

$$
\partial_{\sigma \sigma}^{2} W(\sigma)=C^{-1}(\sigma)
$$

we find:

$$
\Delta W=\phi(\sigma) \Delta \sigma+\frac{1}{2} C^{-1}(\sigma)(\Delta \sigma)^{2} .
$$

This equation has important consequences. First we virtually separate the membrane capacitor into equal patches $I$ and $I I$, each of area $A / 2$, and permit the charge $\Delta q=A \Delta \sigma / 2$ to flow from $I$ to $I I$. For simplicity let us neglect boundary effects and assume that each charge density is uniform $\left(\sigma_{1}=\sigma-\Delta \sigma\right.$ and $\left.\sigma_{2}=\sigma+\Delta \sigma\right)$ and that the patches deform independently, i.e. two membrane capacitors in parallel, not elastically coupled. Since the potential is constant in the plane of the membrane, we find from equation 19 that the total energy change is

$$
\Delta W_{12}=\frac{A}{2} \Delta W_{1}+\frac{A}{2} \Delta W_{2}=\frac{A}{2} C^{-1}(\Delta \sigma)^{2} .
$$

Thus $\Delta W_{12}$ is negative if $C<0$ for the chosen $\sigma$. Put differently, $C<0$ provides a driving force leading to a nonuniform charge distribution and membrane deformation if the artificial $\sigma$-control restrictions are relaxed. 


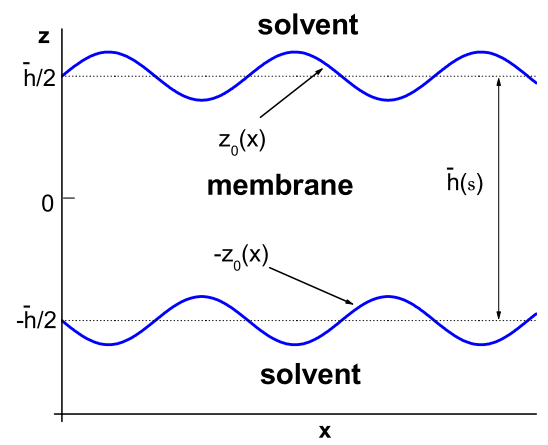

Figure 1. Membrane slab under the effect of symmetric undulations.

The energy penalty results from the continuous transition between the properties of the two membrane patches and can be described as a linear interfacial tension. It is proportional to the length of the border between them and, for large $A$, is negligible relative to $\Delta W_{12}$, equation 20 . Thus this charge density redistribution and the corresponding non-uniform deformation of the membrane is possible energetically for those charge densities that lead to NC assuming $\sigma$-control. The appearance of $C<0$ in treatments that presume a uniform charge density indicates that the system is unstable. This result is similar to the thermodynamic arguments of Nikitas [19] who considered equilibrium conditions between two separate surface phases. We will now show that the prediction of NC under $\sigma$-control indicates the possible formation of the inhomogeneous phase under $q$-control. In our virtual experiment we assumed that the charge density is uniform in each patch. Releasing this restriction provides other pathways for transition to a non-uniform state. Consider, for example, membrane stability relative to symmetric undulations (illustrated in figure 1), the harmonic variation of membrane thickness:

$$
h(x)=\bar{h}+2 u \cos (k x),
$$

where $u$ is the amplitude of the undulation of the membrane surfaces; the corresponding "lower" and "upper" interfaces are described by the equations

$$
z_{u, l}(x)= \pm z_{0}(x), \quad \text { where } \quad z_{0}(x)=\bar{h} / 2+u \cos (k x) .
$$

This problem has been discussed previously for $\phi$-controlled systems (see $[8,23,26]$ and references therein). Now we consider a $q$-control environment and fix the total charge density $q=\sigma A$.

Some aspects of the solution procedure should be stressed.

1. Unlike under $\phi$-control, under $q$-control the transmembrane potential drop $V$ is not fixed by the external source (battery, potentiostat). However, the conductive surfaces are still equipotentials and the potential $\phi_{q}$ is constant on the membrane plane. 
2. The value of $\phi_{q}$ depends on both the original charge density $\sigma$ of the unperturbed membrane and the parameters $u$ and $k$, characterizing the undulations.

3. $\phi_{q}$ is then determined as follows:

(a) The solution for a fixed but arbitrary $\phi$ determines the potential $v(x, z)$ within the membrane $[1,16,23]$.

(b) The equation $\sigma(x)=\varepsilon \varepsilon_{0} \nabla_{n} v\left(x, z_{0}(x)\right)$ determines the local charge density $\sigma(x)$, where $\nabla_{n}$ is the normal derivative taken at the interface $z_{0}(x)$.

(c) The total interfacial charge $\widetilde{q}(V)$, is found by integrating $\sigma(x)$ over the interface with a weighting factor, $1 / \sqrt{1-\left[\partial_{x} z_{0}(x)\right]^{2}}$, that accounts for the membrane stretching associated with undulations.

(d) $\phi_{q}$ is determined from the condition $\widetilde{q}(V)=\sigma A$ :

$$
\phi_{q}=\frac{\sigma}{\varepsilon_{m} \varepsilon_{0}} h\left(1-\frac{k \operatorname{coth}(k h / 2)}{h} u^{2}\right)
$$

with $\bar{h}=\bar{h}(\sigma)=h_{0} \alpha(\sigma)$. The externally fixed potential $\phi$ in the equations for $\phi$-control can now be replaced by $\phi_{q}$, which completes the solution of the problem for $q$-control.

The membrane's electrostatic energy is then

$$
W_{e}^{q}=W_{e, 0}^{q}+W_{e, u}^{q},
$$

where

$$
W_{e, 0}^{q}=\frac{\sigma^{2}}{\varepsilon_{m} \varepsilon_{0}} h(\sigma)
$$

is the energy of the uniform membrane slab and

$$
W_{e, u}^{q}=-W_{e, 0}^{q} \frac{k u^{2}}{\bar{h}(\sigma)} \operatorname{coth}\left[\frac{k \bar{h}(\sigma)}{2}\right]
$$

is the undulatory contribution. The onset of instability is determined by competition between the decrease of the electrostatic energy, equation 24, and the corresponding increase in membrane deformation energy averaged in XY-plane, $W_{d, u}$. For simplicity, we consider small $k$ (the long- wavelength limit) $k h \ll 1$. Similar to [23] where a slightly different form of $W_{d, 0}$, equation 13 , was used, we can represent $W_{d, u}$ as

$$
W_{d, u} \sim \frac{K_{\mathrm{s}} u^{2}}{h_{0}^{2}}\left[1+O\left((k h)^{2}\right)\right]
$$

Higher order terms in $(k h)^{2}$ arise from surface tension and bending contributions to the elastic energy and are neglected. They are analogs to the non-uniform interfacial contributions of the previous example. In the same limit equation 24 can be represented as

$$
W_{e, u}^{q} \sim-2 W_{e, 0}^{q} u^{2}\left[\frac{1}{\bar{h}(\sigma)^{2}}+O\left((k \bar{h})^{2}\right)\right] .
$$


The uniform distribution becomes unstable when

$$
W_{u}=W_{d, u}+W_{e, u}^{q} \leqslant 0 .
$$

Substituting equations 24 and 14 we find the condition of instability:

$$
|\bar{\sigma}| \geqslant \sigma_{\mathrm{cr}}
$$

which is equivalent to

$$
C_{q}^{-1} \leqslant 0
$$

Our thought experiment presumed that the conditions for $\sigma$-control, i.e. uniformity of $\sigma$, could be arbitrarily relaxed anywhere within the negative $C$ domain. In reality there is no way to enforce uniformity when the system is unstable; thus the distribution spontaneously becomes inhomogeneous at the edge of this domain with critical point $\sigma=\sigma_{\mathrm{cr}}$, where

$$
C_{q}^{-1}\left(\sigma_{\mathrm{cr}}\right)=0
$$

the transition actually occurs a bit earlier, at a point roughly determined by a Maxwell construction [27].

We have analyzed a simplified model corresponding to a concentrated electrolyte (Debye length $\lambda_{\mathrm{D}} \rightarrow 0$ ). Further analysis based on our previous work shows that equation 27 also determines the onset of instability for finite $\lambda_{\mathrm{D}}$ [23]. This result is valuable on its own. As already indicated the Poisson-Boltzmann approximation and other local statistical models do not predict NC (see $[10,27,28]$ and references therein). Equations 11 and 12 show that for these models the rate of gap contraction with the charging, $d^{\prime}(\sigma)$, is always less than $1 /|\sigma|$ and thus $C_{\sigma}$ is always positive. Consequently, this class of models would not satisfy the criteria suggested in [11] linking the model quality to the appearance of a $C_{\sigma}<0$ domain. Our results indicate that adding another relaxation mechanism immediately leads to the appearance of a $C_{\sigma}<0$ domain and instability. This further illustrates that anomalies are more typical than expected based on purely ionic models with immobile charged plates.

In previous analysis [22] we considered two elastically coupled membrane capacitors, with the extra term in the deformational energy $\sim \alpha\left(u_{1}-u_{2}\right)^{2}$ accounting for the non-uniformity penalty (differential thinning of the patches). Depending on the coupling constant $\alpha$, this system could exhibit NC before transition to a nonuniform state. Our present discussion implies that such a picture is unrealistic. In terms of the first model, the constant $\alpha$ must be proportional to the width of the transition region relative to the area of the patches $A$ and thus can become infinitesimal if $A$ is sufficiently large. In addition, as shown in the second example, the non-uniformity contributions vanish at small $k$ which provides a reasonable pathway for the onset of instability.

Finally, we reiterate the major difference between $q$ - and $\phi$-control for the onset of instability. Under $\phi$-control (an open system connected to a potentiostat) the stability is lost simultaneously for both uniform deformation and undulations [8, 23]. In contrast, a $q$-controlled (isolated) system is always stable with respect to uniform deformation (see equation 18 and the corresponding discussion) and only loses stability in making a transit to a nonuniform state. 


\section{Perspectives for further study}

It is by now well established that the capacitance can be negative for uniformly charged surfaces under the artificial conditions of $\sigma$-control. Many statistical ionic models have demonstrated this anomaly (see $[6,11,31]$ and references therein). While in the 1980s many would have viewed such predictions as model faults, the most recent view $[6,11]$ represents a dramatic change of mind, and it is even suggested that the prediction of $C<0$ must be considered as a criterion validating an ionic model [11] rather than an imperfection. We do not disagree. In fact, it accords with our observation $[10,13,22,27]$ that such predictions are typical of models jointly accounting for various contributions (electronic, ionic, etc.) to the charging induced relaxation of the effective gap of the interfacial capacitor.

What is the physical significance of such predictions? Our analysis indicates that they imply an instability with respect to a transition to an inhomogeneous state. To analyze the consequences, the artificial assumption of a uniform surface charge distribution must be dropped, given that in a real metallic electrodes the electrons are free to move and thus surface charge density can become, at least in principle, laterally non-uniform. This non-uniformity can be even more pronounced in soft media such as lipid bilayers. The value of the models predicting this anomaly, emphasized in [11], is even greater since they are candidates for analyzing such an instability.

We must re-emphasize that in our usage the terms "instability" and "transition" are not related to real interfacial critical phenomena. While the phase transition actually occurs before $C_{\sigma}$ becomes negative, we permit the system to enter this domain by artificially maintaining $\sigma$-control. Relaxing the uniformity constraint at any $\sigma$ within the $\mathrm{NC}$ region leads immediately to a transition to an inhomogeneous state of fixed $q, q=\sigma A$. Although the initial ( $\sigma$-controlled) state is artificial, the final stable inhomogeneous phase (if it exists) is real since the equilibrium state is unique. Thus, our approach is useful for testing and developing statistical models that describe both uniform $(C>0)$ and inhomogeneous (regions with $C<0$ under $\sigma$-control) phases.

Predicting instability does not necessarily imply that the model describes the formation of a new stable inhomogeneous phase. As the transition can be accompanied by a substantial local increase of the charge density and a corresponding local increase of the ionic density in the EDL, a model must have a stabilization mechanism that interrupts the propagation of instability. This would permit the formation of a new stable phase before the condition of "ideal polarizability" is broken and interfacial charge transfer occurs. Ionic size and correlation effects in the electrolyte should be of importance here.

Although the condition $C<0$, obtained for a primitive ionic model of the EDL, can be an important factor leading to instability, it is not a necessary condition. Even with ionic models that by themselves do not lead to instability (such as GCS model) the addition of other mechanisms of relaxation, such as a displacement of the "electronic plate" of the interfacial capacitor, can lead to $C_{\sigma}<0$ and thus 
trigger the instability $[10,22,28]$. In other words, this anomaly should be even more commonplace than is implied by ionic model studies.

Another important question is a comparison of phase transitions predicted for the open and the isolated systems. While in the first case the transition can be accompanied by charging the electrodes, in the second case the lateral variation of charge keeps the total charge fixed. Finally, the inherent inhomogeneity (roughness) of an electrode surface (especially with respect to solid electrodes) must be considered. The effect of the roughness on the equilibrium properties of EDL is well established [17], and its possible effect on the surface phase transition can also be a promising field for further research.

\section{Acknowledgement}

Work supported by a grant from the National Institutes of Health, GM28643. M. B. P. wishes to thank Dr. V.J.Feldman for many fruitful discussions and Professor A.M. Brodsky for suggesting the possibility of charge density-type phases in double layers (private communication, $\sim 1988$ ).

\section{References}

1. Andelman D.. Electrostatic properties of membranes: The Poisson-Boltzmann theory, Lipowsky R., Sackmann E., editors. Handbook of Biological Physics, vol. 1, chapter 12, p. 603-642. Elsevier Science, Washigton, DC., 1995.

2. Attard P., Wei D., Patey G.N., On the existence of exact conditions in the theory of the electrical double layer. J. Chem. Phys., 1992, 96, 3767-3771.

3. Blum L., Theory of electrified interfaces. J. Phys. Chem., 1977, 81, 136-147.

4. Blum L., Lebovitz J.L., Henderson D., A condition on the derivative of the potential in the primitive model of an electric double layer. J. Chem Phys., 1980, 72, 4249-4250.

5. Bockris J.M., Reddy A.K.N.A., Gamboa-Aldeco M. Modern Electrochemistry: Fundamentals of Electrodics, $2^{\text {nd }}$ edition. Plenum Publishing Corporation, New York, 2000.

6. Boda D., Henderson D., Plaschenko P., Fawcett W.R., Monte Carlo and density functional theory study of the electrical double layer: the dependence of the charge/voltage relation on the diameter of the ions. Molecular Simulation, 2004, 30, 137-141.

7. Cooper I.L., Harrison J.A., Some observations on the inner layer at the mercuryelectrolyte interface. J. Electroanal. Chem., 1975, 66, 85-98.

8. Crowley J.M., Electrical breakdown of bimolecular lipid membranes as an electromechanical instability. Biophys. J., 1973, 13, 711-724.

9. Feldman V.J., Partenskii M.B., Vorobjev M.M., Density functional approach to the metal-solid electrolyte interface: Electron relaxation effect, equilibrium electrical properties and bilayer instability problem. Electrochim. Acta., 1986, 31, 291-297.

10. Feldman V.J., Partenskii M.B., Vorobjev M.M., Surface electron screening theory and its applications to metal-electrolyte interface. Prog. Surf. Sci., 1986, 23, 3-154.

11. Gonzalez-Tovar E., Jimenez-Angeles F., Messina R., Lozada-Cassou M. A new correlation effect in the Helmholtz and surface potentials of the electrical double layer. J. Chem. Phys., 2004, 120, 9782-9792. 
12. Henderson D., Blum L., Smith W.R., Application of the hypernetted chain approximation to the electric double layer at a charged planar interface. Chem. Phys. Let., 1979, 63, 381-388.

13. Kim Z.B., Kornyshev A.A., Partenskii M.B., On the anomalously high and negative values of the compact layer capacity in some new models of the metal/electrolyte interface. J. Electroanal. Chem., 1989, 265, 1-9.

14. Landau L.D., Lifshitz E.M. Electrodynamics of Continuous Media. Pergamon Press, Oxford, 1960.

15. Landau L.D., Lifshitz E.M. Theory of Elasticity. Butterworth-Heinemann, Oxford, 1970.

16. Leikin S., Thermal fluctuations and capacitance of bilayer lipid membranes in electric field. Biologicheskie Membrani, 1985, 2(8), 820-831 (in Russian).

17. Daikhin L.I., Kornyshev A.A., Urbakh M., Double-layer capacitance on a rough metal surface. Physical Review E, 1996, 53, 6192-6199.

18. McCombie C.W. Fluctuations of general classical mechanical variables (problem 21.3), Landsberg P.T., editor. Problems in Thermodynamics and Statistical Physic, p. 459. Pion, London, 1971.

19. Nikitas P., Stability conditions at charged interfaces. J. Electroanal. Chem., 1991, 316, 23-35.

20. Nikitas P., Phase transitions in the multilayer model of a charged interface and the problem of negative capacitance. Electrochim. Acta, 1992, 37, 81-90.

21. Nikitas P., Anastopolous A., Papanastasiou G., Phase transitions in models for adsorption on electrodes. Further examination of polarization catastrophe. J. Electroanal. Chem., 1991, 317, 43-76.

22. Partenskii M.B., Dorman V.L., Jordan P.C., The question of negative capacitance and its relation to instabilities and phase transitions at electrified interfaces. Int. Rev. Phys. Chem., 1996, 11, 153-181.

23. Partenskii M.B., Dorman V.L., Jordan P.C., Membrane stability under electrical stress: A non-local electroelastic treatment. J. Chem. Phys., 1998, 109, 10361-10371.

24. Partenskii M.B., Feldman V.J., Electron and molecular effects in the double-layer for the metal electrolyte solution interface. J. Electroanal. Chem., 1989, 84, 57-68.

25. Partenskii M.B., Jordan P.C., Membrane capacitance: Nonlocal electroelastic treatment. Mol. Phys., 2000, 98, 193-200.

26. Partenskii M.B., Jordan P.C. Electroelastic instabilities in double layers and membranes, Volkov A.G., editor. Liquid interfaces in chemical, biological, and pharmaceutical applications, vol. 95 of Surfactant Science Series, chap. 3, p. 51-82. Marcel Dekker, Inc., New York, 2001.

27. Partenskii M.B., Jordan P.C., The admissible sign of the differential capacity, instabilities, and phase transitions at electrified interfaces. J. Chem. Phys., 2002, 117, 2992-3002.

28. Partenskii M.B., Jordan P.C. Negative capacitance and related instabilities in theoretical models of the electric double layer and membrane capacitors.

Preprint arXiv: physics/0412183, 2005.

29. Partenskii M.B., Kim V.J., Feldman Z.B., Anomalous electric properties and stability of a double-layer at the boundary metal electrolyte - capacity of a double-layer with relaxing plates. Sov. Phys. J., 1987, 30, 907-913.

30. Schmickler W., Henderson D., New models for structure of the electrochemical inter- 
face. Prog. Surf. Sci., 1986, 22, 323-419.

31. Torrie G.M., Negative differential capacities in electrical double-layers. J. Chem. Phys., 1992, 96, 3772-3774.

32. Wei D., Torrie G., Patey G., Molecular solvent model for an electrical double layer: Effects of ionic polarizability. J. Chem. Phys., 1993, 99, 3990-3997.

\title{
Негативна ємність та нестабільність біля заряджених поверхонь: уроки, що випливають 3 вивчення ємності мембран
}

\author{
М.Б.Партенський, П.К.Джордан \\ Відділення хімії університету Брендіз, \\ Волтхем, США
}

Отримано 14 вересня 2004 р.

Представлено короткий огляд моделей, які передбачають негативну ємність $C$. Обговорюється роль цих моделей у явищі електричного контролю. Ми ще раз показуємо, що розрахункова ємність подвійного шару може бути негативною завдяки $\sigma$-контролю - штучній конструкції, яка вимагає однорідного розподілу густини поверхневого заряду електрода, $\sigma$. Разом з тим, тільки загальний заряд $\boldsymbol{q}$ (або усереднена густина поверхневого заряду $\bar{\sigma}$ ) може бути експериментально зафіксованою при дослідженні ізольованої комірки ( $q$-контроль), Для значень $\sigma$, де $C$ стає від'ємною в умовах $\sigma$-контролю, перехід до $\boldsymbol{q}$-контролю (тобто релаксація латеральної густини розподілу заряду шляхом фіксації ії середнього значення до величини $\sigma$ ) веде до нестабільності однорідного розподілу і переходу до неоднорідної фази. В якості ілюстрації розглядається модель "мембранного конденсатора". Ця точно розв'язувана модель допускає як однорідну, так і неоднорідну релаксацію і таким чином допомагає продемонструвати зародження і деякі важливі риси нестабільності. Коротко обговорюються можливості подальших досліджень.

Ключові слова: електрохімічні інтерфейси, нестабільність та фазові переходи, електронний подвійний шар, ємність

PACS: 68.35.Rh, 68.35.Md, 82.45.Mp, 82.45.Rr, 82.45.Uv, 68.08.-p, 73.30.+y 